\title{
Two-Phase System Approach as Standerless Procedure for Crystallinity Determination Using Rietveld Method
}

\author{
A. A. Ramadan ${ }^{1)}$, M. R. Ebeid ${ }^{2)}$, S. K. Abdelraheem ${ }^{2)}$ and \\ E. M. Abdel-Minem ${ }^{2)}$ \\ 1) Physics Department, Helwan University, Helwan, Cairo, Egypt \\ ${ }^{2)}$ Physics Department, Minia University, Minia, Egypt
}

The two-phase system approach is a method to determine the degree of crystallinity without a need for a stander. The method applies the crystallite theory of amorphous models and uses the great potentiality of Rietveld analysis of multiphase systems considering the crystalline and amorphous counterpart as two different phases. It was verified, first, by artificial mixtures using simulated X-ray diffraction patterns and then tested by experimental diffraction patterns obtained from synthetic mixtures of crystalline and amorphous $\mathrm{SiO}_{2}$ with different ratios. The models for the crystalline and crystalline counterpart of the amorphous phases were, respectively, considered to have the hexagonal space group $\left(\mathrm{P}_{3}{ }_{2}\right)$ and the cubic space group $\left(\mathrm{P} 2{ }_{1} 3\right)$. The proposed approach was used for the determination of the degree of crystallinity and can be considered as standarless method free from the effect of the matrix absorption and the improbable background estimation. Also, higher accuracy was achieved comparing with those calculated by using crystalline index method. In addition to crystallinity determination, the method gives a useful structural picture of crystalline and amorphous counterparts at the same time.

\section{Introduction:}

Study of the materials in the amorphous state and information about the amorphous content in a crystalline matrix are of great importance from the scientific and technological points of view. More over, the structural information about the amorphous materials and the crystalline-to-amorphous ratio (crystallinity) are essential prerequisites to understand the physical and chemical properties of the considered materials. Unlike the crystalline solids, the structure of even the simplest single component glasses is still under debate. One of the theories of the amorphous models is the crystallite theory, which envisaged glass as an assembly of very small crystals (crystallites) of the same structure as the corresponding crystalline counterpart [1]. Thus, useful 
information on the structure of amorphous solids can be obtained from a simple comparison with the corresponding crystalline polymorphs as a starting point for structural models. This does not necessarily mean that the crystallite theory is accepted, but the short-range order may resemble that of the associated crystals.

In order to obtain the correct form of diffraction pattern for the amorphous phase from a crystal based model, it is necessary to include disorder (strain). Le Bail et al. [2] and Le Bail [3] have applied a modified Rietveld program (ARITVE) to refine pure amorphous phases like crystalline ones using microstrained crystalline models. This is based on the fact that, when a crystalline model is distorted mathematically by the application of a statistical isotropic microstrain and/or decreasing the crystallite size (nano-crystallite), an artificial amorphous-like powder diffraction patterns are produced. The method was considered [4] as a variation of the Reverse Monte Carlo (RMC) technique. On the other hand, there are problems in the determination of the degree of crystallinity by X-ray diffraction (XRD); a major one is the correct separation of the sharp peaks from the continuous scattering (amorphous and background contribution). Fortunately, it has become clear that Rietveld method can provide very accurate estimates of the relative and/or absolute abundance of the components of multiphase samples [5]. Thus, combining the suggestion presented by the crystal based model and the advantage of Rietveld method in quantitative phase analysis seems to be of great help in the problem of crystallinity determination. The present work was achieved [6] and presented by Ramadan et al. [7] considering the effect of both the small size of the crystallites (nanocrystallite) and the internal microstrain. However, Lutterotti et al. [8] investigated the same problem in ceramic materials but have used only the concept of small grain size.

In the present paper, the suggestion offered by the crystallite theory is combined with the advantage of Reitveld method in quantitative phase analysis to apply a proposed two-phase system approach for crystallinity determination. It is based on a study to verify the crystal based model using the effect of both the crystallite size and the microstrain $\left[\begin{array}{lll}6 & \& & 9\end{array}\right]$. In order to avoid the experimental errors and ambiguity, the prominent features of the computerassisted physics was considered as a tool in the present work and simulated data will be, first, considered.

\section{Experimental:}

The starting (raw) materials were pure crystalline $\mathrm{SiO}_{2}\left(\alpha_{\text {-quartz}}\right)$ and vitreous silica (amporphous) with purity of $99.9 \%$. Mixtures were synthesized with different crystalline-to-amorphous ratios (80:20, 50:50 and 20:80). The samples were carefully grounded in agate mortar to get a homogenous mixture. 
XRD patterns of the "synthetic" mixtures as well as the pure crystalline and pure amorphous forms of $\mathrm{SiO} 2$ were obtained by Siemens D5000 Diffractometer. Ni-filtered $\mathrm{Cu} K^{\alpha}$ radiation was used at high stabilized power of $40 \mathrm{kV}$ and $30 \mathrm{~mA}$. Considerable number of counts were collected using stepscanning mode in $2 \theta$-range from 10 to $100^{\circ}$ with step size of $0.05^{\circ}(2 \theta)$ and the sampling time at each step of $10 \mathrm{~s}$.

\section{Methods:}

\subsection{Structure refinement by Rietveld method:}

The LS1 computer program [10] was used for Rietveld analysis as well as simulation of diffraction patterns. The program has introduced profile analysis into the Rietveld method. The devised algorithm simultaneously refines the structural parameters as well as the micro-structural characteristics of samples (internal microstrain and crystallite size parameters). The other parameters are scale factors, crystallographic parameters (cell parameters, atomic displacements, atomic occupation numbers, temperature factors, preferred orientation parameters) and background coefficients. The final refinable parameters were accepted according to the Rietveld weight profile reliability, $\mathrm{R}_{\mathrm{wp}}$;

$$
\mathrm{R}_{\mathrm{wp}}=\sqrt{\frac{\sum \mathrm{w}_{\mathrm{i}}\left(\mathrm{Y}_{\mathrm{ci}}-\mathrm{Y}_{\mathrm{oi}}\right)^{2}}{\sum \mathrm{w}_{\mathrm{i}}\left(\mathrm{Y}_{\mathrm{oi}}\right)^{2}}}
$$

where $\mathrm{Y}_{\mathrm{Oi}}$ and $\mathrm{Y}_{\mathrm{ci}}$ are the observed and calculated intensity at the $\mathrm{i}^{\text {th }}$ step while $\mathrm{w}_{\mathrm{i}}$ is the weighting factor $=1 / \mathrm{y}_{\mathrm{oi}}$.

\subsection{Models of amorphous and crystalline Silica:}

Due to the similarities in bonding, density and position of the first diffraction peak of $\beta$-cristbolite and amorphous silica, Le Bail [3] and Gaskell and Wallis [11] suggested that there is a close structural relationship over short length scales between the two phases. Le Bail [3] selected this model on the basis of highest level of agreement among all the tested cases according to the conventional Rietveld [12] profile reliability $R_{p}$ factor. Lowering the symmetry from the cubic space group $\mathrm{P} 2{ }_{1} 3$ (\# 198, International Table) to the orthorhombic $\mathrm{P} 2{ }_{1} 2_{1} 2_{1}$ (\# 19, International Table) lead only to a small improvement. Using Rietveld analysis [12] and Reverse Monte Carlo refinement [13 \& 14], the detailed quantitative study [15] showed that the similarities between the crystalline ( $\beta$-cristbolite) and amorphous (silica) 
phases extends over a correlation length of $0.75 \mathrm{~nm}$. Thus, one can say that up to this length scale there are regions of the glass that have the structure elements of the crystal, and beyond this distance the differences between crystal and glass diverge.

In this work, the simple cubic structure of high $\beta$-cristbolite was the considered as the crystalline-based starting model, as recommended [3], to produce the artificial amorphous-like powder (randomly oriented polycrystallites) diffraction patterns. The used model [16] has a lattice parameters $\mathrm{a}=0.7160 \mathrm{~nm}$ and number of molecules per unit cell $\mathrm{z}=8$. The eight silicon and four of the oxygen atoms are placed at positions of (4a): with $\mathrm{u}(\mathrm{Si}, 1)=0.255, \mathrm{u}(\mathrm{Si}, 2)=-0.008$ and $\mathrm{u}(\mathrm{O})=0.125$. The other twelve oxygen atoms are at the positions of (12b): with $\mathrm{x}=\mathrm{y}=0.66$ and $\mathrm{z}=0.06$. With crystallographic information and increasing microstrain up to 0.08 and decreasing crystallite size down to about $10 \mathrm{~nm}$, the simulated diffraction pattern of the amorphous phase was obtained as shown in Fig.(1). From the simulated intensities, the one dimension radial distribution function, RDF, and the real space correlation function, $\mathrm{T}(\mathrm{r})$, have been calculated obeying the line of Wright [1]. The obtained curves consists of three peaks in short range region at $\mathrm{r}=0.16,0.26$ and $0.31 \mathrm{~nm}$ as shown in Fig.(2). Comparing these values and simulated curves with those given by Wright [1], the simulation pattern can be safely accepted.

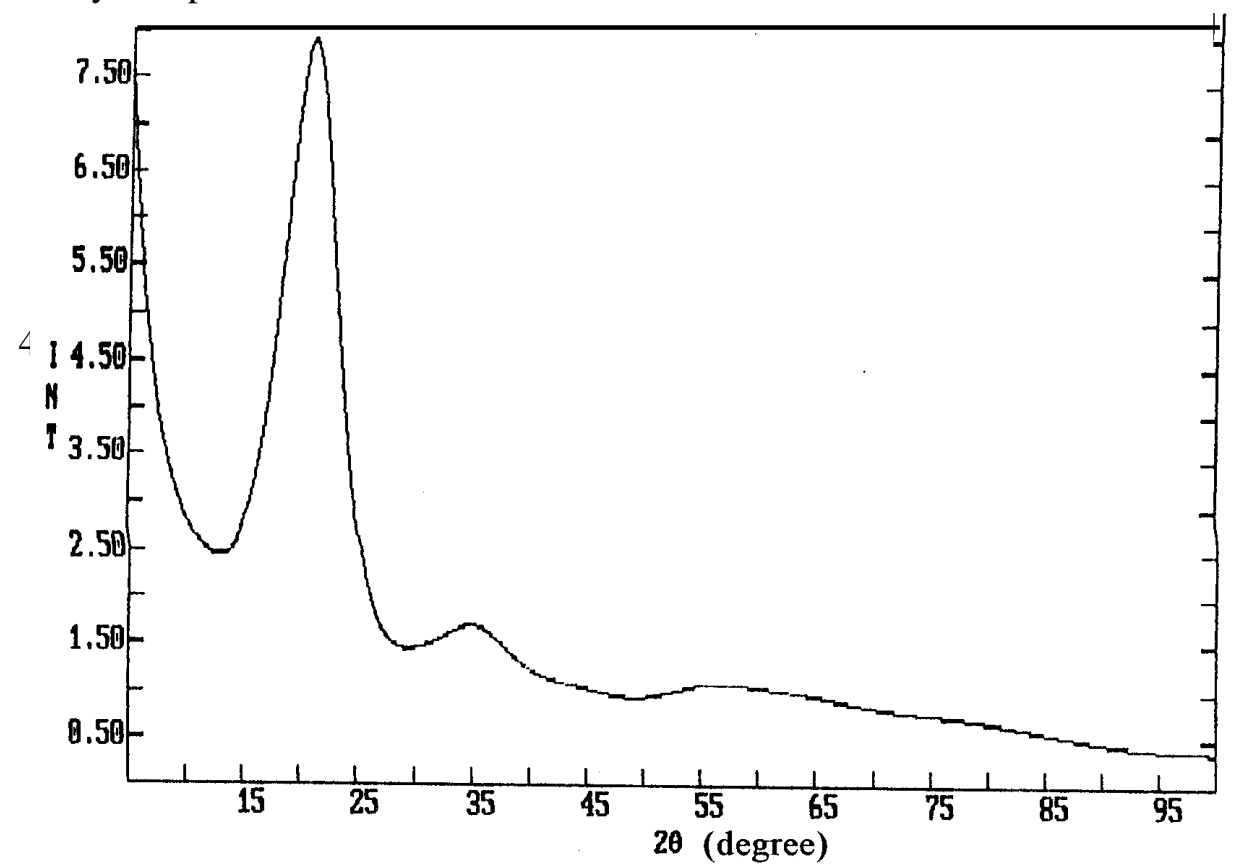

Fig.(1): Simulated (artificial) diffraction pattern of amorphous $\mathrm{SiO}_{2}$. 

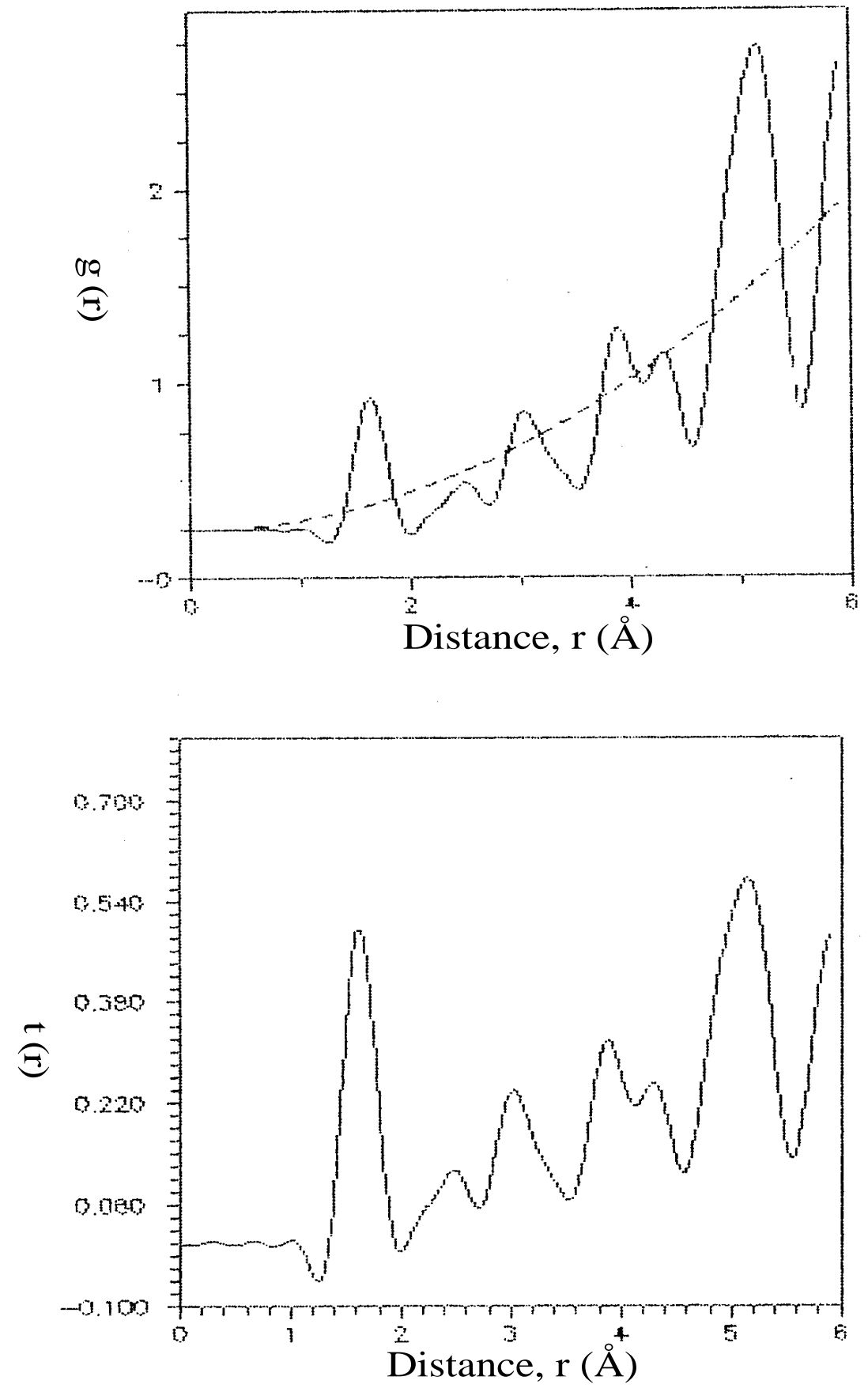

Fig.(2): a) Radial distribution and b) correlation function curves of simulated (artificial) amorphous $\mathrm{SiO}_{2}$. 
The hexagonal structure of low temperature $\alpha$-quartz [16] of space group $\mathrm{P}_{1}{ }_{2}$ (\# 151, International Table) has been used to represent the crystalline model of $\mathrm{SiO}_{2}$ with lattice parameters: $\mathrm{a}=0.4913 \mathrm{~nm}, \mathrm{c}=0.5405$ $\mathrm{nm}$, number of molecules per unit cell, $\mathrm{Z}=3$. The Si-atoms are placed in three positions (3a): with $\mathrm{u}=0.465$. The $\mathrm{O}$-atoms are in six positions $(6 \mathrm{c})$ : with $\mathrm{x}=$ $0.415, \mathrm{y}=0.272$ and $\mathrm{z}=0.120$. Using the parameters of this model and low microstrain down to 0.0001 large crystallite size up to $500 \mathrm{~nm}$, the simulated diffraction pattern typical for the crystalline phase was obtained.

\subsection{Two-phase System Approach:}

The proposed two-phase system approach for crystallinity determination is based on the crystallite theory and the technique of Rietveld quantitative phase analysis. This technique has been demonstrated [5] that there is a simple relationship between the scale factors determined in Rietveld analysis and the phase composition of a multicomponent mixture. The sample has been considered as a mixture of two phases; one is crystalline and the other is amorphous. Structural characteristics for the models of the crystalline and crystalline counterpart of the amorphous phases are given above. The parameters (scale factor, lattice constants, microstrain and crystallite size) are all refined for each phase without any constraint by the codewords of the same parameter, i.e. not assigning the same codeword to the same structure parameter in the two phases. The weight fraction of crystalline phase (the degree of crystallinity), $\mathrm{wt}_{\mathrm{c}}$, is given as:

$$
\mathrm{wt}_{\mathrm{c}}=\frac{(\mathrm{SZMV})_{\mathrm{c}}}{(\mathrm{SZMV})_{\mathrm{c}}+(\mathrm{SZMV})_{\mathrm{a}}}
$$

where S, Z, M and V are, respectively, Rietveld scale factor, the number of the formula units per unit cell, the mass of the formula unit and unit cell volume. The letters a and c stand for amorphous and crystalline phases, respectively.

For comparison, the crystalline index, using the method of Wokllin et al. [17], is determined as one of the most used method for degree of crystallinity determination. In this method the integral form of crystalline index, $\chi$, is given as [18]:

$$
\chi=\frac{\sum\left(\mathrm{I}_{\mathrm{s}}-\mathrm{I}_{\mathrm{a}}\right)_{\mathrm{i}}}{\sum\left(\mathrm{I}_{\mathrm{c}}-\mathrm{I}_{\mathrm{a}}\right)_{\mathrm{i}}}
$$


where $I_{a}, I_{c}$ and $I_{S}$ are, respectively, the diffraction intensities of pure, crystalline and mixture sample recorded at the same Bragg angle $2 \theta_{\mathrm{i}}$. Summation is carried out over the whole diffraction pattern.

\section{Results and Discussion}

In order to avoid the experimental errors and ambiguity, a simulated (artificial) data was, first, considered then the experimental (synthetic) one has been used.

\subsection{Artificial mixtures}

The XRD patterns for artificial samples of different degree of crystallinities were calculated by mixing the intensities of the simulated crystalline and amorphous diffraction patterns at each $2 \theta$-step to ratios of 95:5, 90:10, 80:20, 50:50, 20:80, 10:90 and 5:95. Before verification of the proposed method, the crystalline index, $\chi$, of the different artificial mixtures is calculated using Eqn.(3). The obtained results and the actual degree of crystallinity, $\mathrm{wt}_{\mathrm{ac}} \%$, which are the weight fraction of crystalline contents are given in Table(1). A high agreement between the actual crystallinity, $\mathrm{wt}_{\mathrm{ac}} \%$, and the calculated crystallinity index $\chi$ was achieved.

Table(1): Actual and calculated crystallinity as well as the crystallinity index of the "artificial" mixtures.

\begin{tabular}{||c|c|c||}
\hline $\mathrm{wt}_{\mathrm{ac}}(\%)$ & $\mathrm{wt}_{\mathrm{c}}(\%)$ & $\chi(\%)$ \\
\hline \hline 95.0 & 94.9 & 95.0 \\
90.0 & 89.9 & 90.0 \\
80.0 & 79.9 & 80.0 \\
50.0 & 49.9 & 50.0 \\
20.0 & 20.1 & 20.0 \\
10.0 & 10.1 & 10.0 \\
5.0 & 5.2 & 5.0 \\
\hline
\end{tabular}

At this step, the proposed method has to be verified using the same simulated mixtures given above. First, the crystallographic and micro-structural parameters are taken equal to those in the models used for simulation and were kept fixed, while only the scale factor was taken far from the real value and 
left free. After few iterations, the refinement was stopped and reached to best fitting for the all samples with $\mathrm{R}_{\mathrm{wp}}$ less than 0.001. The artificial intensities, the fitting curve and the differences (residuals) between artificial and fitting intensities for the sample of $20 \%$ crystalline phase (as an example) are depicted in Fig.(3). From the obtained values of the scale factor and using Eqn.(2), the crystallinity, $\mathrm{wt}_{\mathrm{c}} \%$, is calculated and listed in Table(1). The good agreement is very clear, which was achieved very easily in this case.

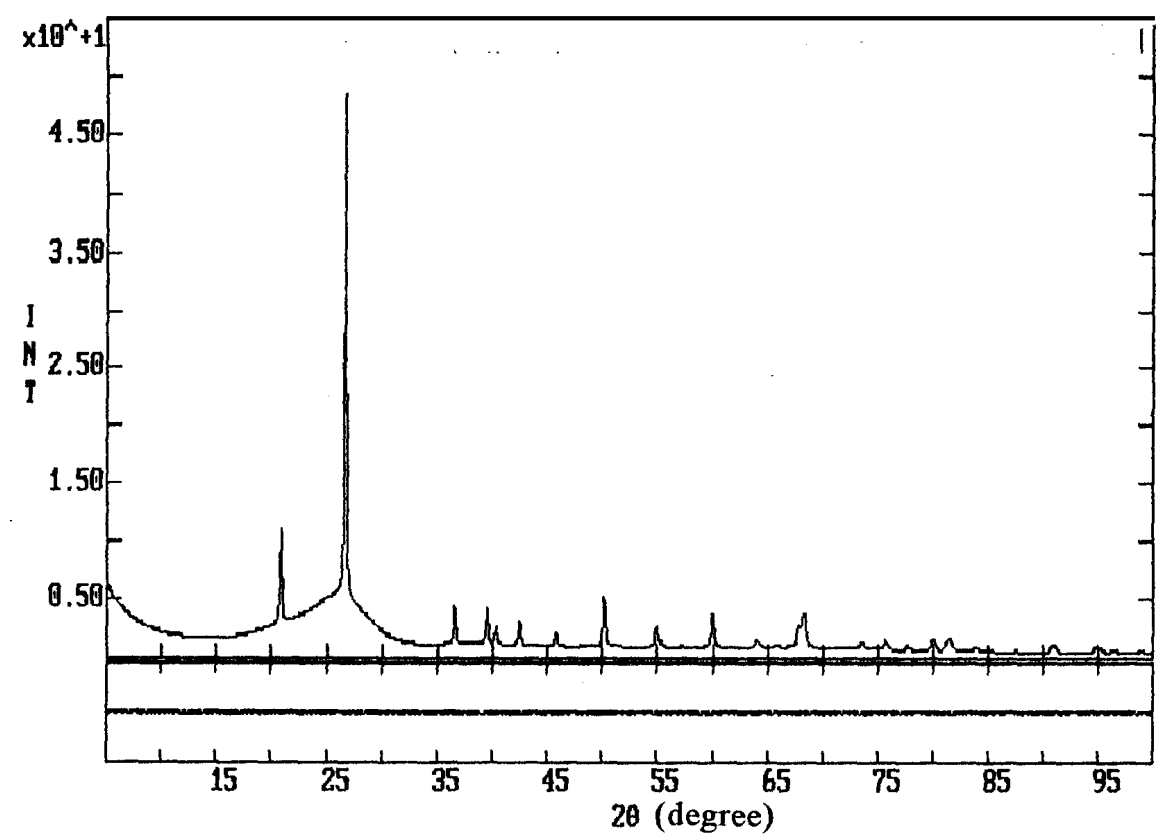

Fig.(3): Rietveld refinement plot for the simulated (artificial) mixture of 20\% crystalline phase.

Now, the verification of the proposed method has to go a step further into the refinement process by adding other structural parameters of the two models for crystalline and amorphous phases. In this case, all parameters will be refined, simultaneously, starting with values smaller or greater than that of the models. The number of iterations for best fitting depends on how the values of the starting parameter are far from the model ones. An acceptable values for all refined parameters were achieved, with less than $1.5 \%$ deviation from the actual values. The calculated degrees of crystallinity do not have any significant differences with those in Table (1). So far, the stability of program has been checked out and a good picture of structural parameters can be obtained using this approach refinement technique. 


\subsection{Synthetic mixture}

After the proposed method has been tested by the simulated data, it was applied to the experimental XRD pattern of synthetic mixtures. The data obtained is investigated by the proposed method. The background was refined using a second polynomial. The scale factors, crystallographic parameters and micro-structural parameters (the crystallite size (L) and microstrain (e)) were refined simultaneously.

The refinements for the mixtures show that the crystalline and amorphous phases have the crystallographic parameters of the model that used in the corresponding single phase, except the values of scale factor. The reliability values were $\mathrm{R}_{\mathrm{wp}} \cong 0.10-0.12$. The Rietveld refinement plot for sample of $20 \%$ crystalline $\mathrm{SiO}_{2}$ is depicted in Fig. (4) as an example. Values of $\mathrm{wt}_{\mathrm{c}}$ and $\chi$ were calculated by Eqn.(2) and Eqn.(3), respectively, and they are given in Table (2) with the actual degree of crystallinity for the same samples.

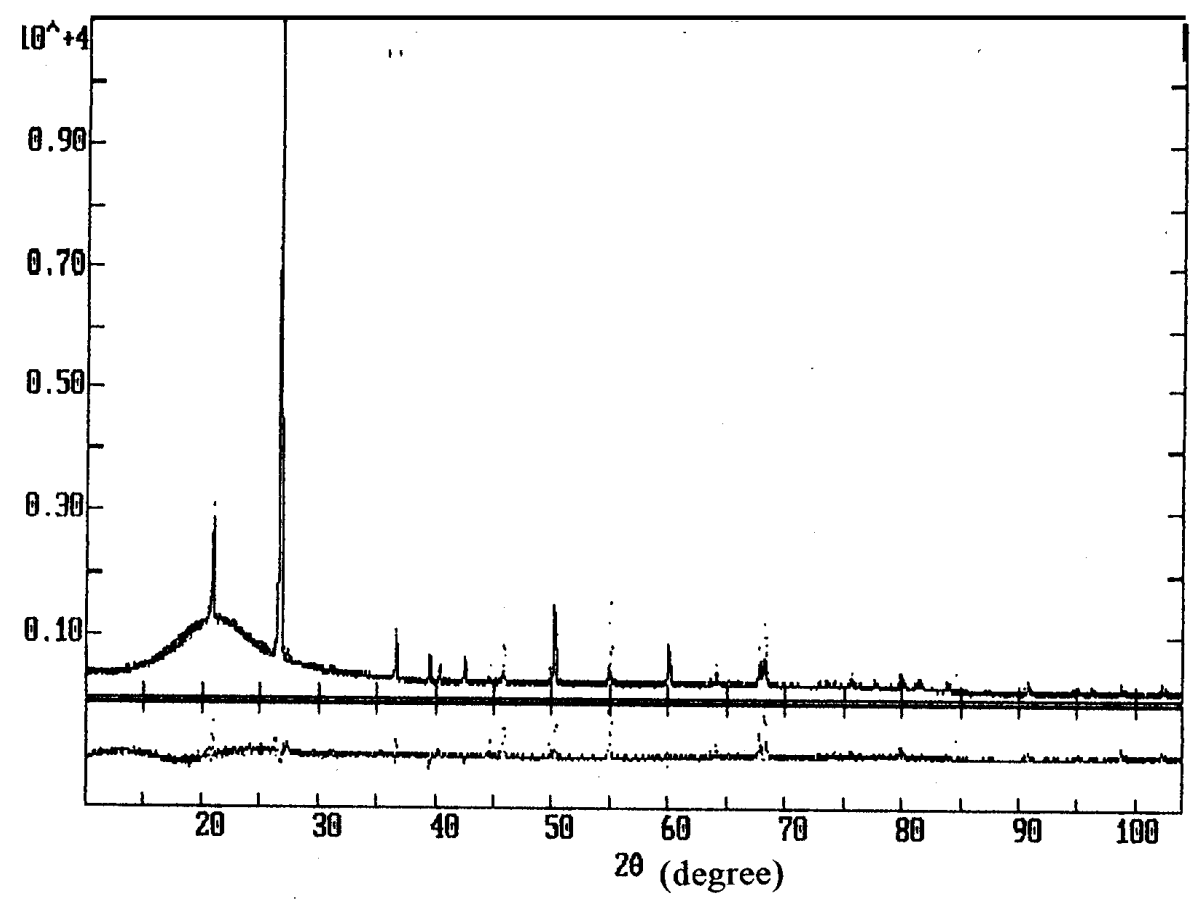

Fig.(4): Rietveld refinement plot for the experimental (synthetic) mixture of $20 \%$ crystalline phase. 
Table(2): Actual and calculated crystallinity as well as the crystallinity index of the "synthetic" mixtures.

\begin{tabular}{|c|c|c|}
\hline $\mathrm{wt}_{\mathrm{ac}}(\%)$ & $\mathrm{wt}_{\mathrm{c}}(\%)$ & $(\%) \chi$ \\
\hline \hline 80 & 79.7 & 62.5 \\
50 & 50.3 & 40.1 \\
20 & 17.0 & 12.9 \\
\hline
\end{tabular}

A plot of the actual degree of crystallinity, $\mathrm{wt}_{\mathrm{ac}} \%$, versus the calculated one, $\mathrm{wt}_{\mathrm{c}} \%$, and crystalline index, $\chi$, is depicted in Fig. (5). It is clear that, in case of $\chi$ the relation deviates from linearity. It shows a curvature, where the calculated $\chi$ is always less than the actual experimentally specified value, i.e., underestimated. On the other hand, it is worth mentioning that the relation between the actual values and that calculated by the proposed method is a straight line with correlation coefficient of 0.99 .

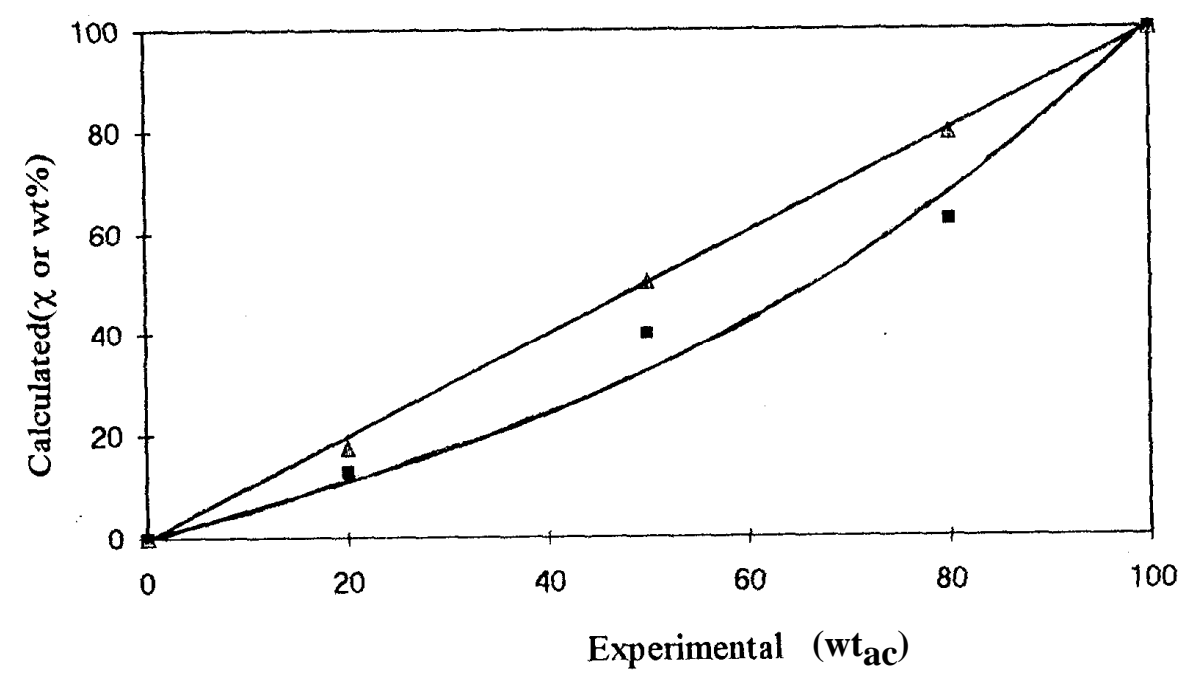

Fig.(5): Plot of the calculated crystallinities $\left(\mathrm{wt}_{\mathrm{c}} \& \mathcal{X}\right)$ vs the actual experimental one $\left(\mathrm{wt}_{\mathrm{ac}}\right)$ for the synthetic samples. [ $\left.\mathrm{wt}_{\mathrm{c}}: \boldsymbol{\Delta} \& \mathcal{\chi}: \mathbf{\square}\right]$.

Usually, deviation from linearity in quantitative analysis is due to the difference in mass absorption coefficient of the present phases. However, the non-linear behavior of the relation shown in Fig.(5) in the case of $\chi$ does not seem to be due to the difference in the mass absorption coefficients of the crystalline and amorphous content. This is because the mass absorption 
coefficient is calculated from the weight fraction and the absorption for the individual element in the compound. Thus, it is independent of the physical state (crystalline or amorphous and even liquid or gas) [19]. The expected main effect in this case is the improper choice of the background; either for the standards or for the samples. However, it is clear that the proposed method is free from either the effect of matrix absorption and/or background estimation.

\section{Conclusions:}

The proposed two-phase system approach, using the suggestion of the crystal theory of amorphous structure and the quantitative phase analysis possibility of Rietveld method, can be used as a "standardless" method for degree of crystallinity determination free from the effect of the matrix absorption and background estimation. The two-phase system method has all the advantages of full-pattern analysis; the background is properly defined, all reflections are included, thus, the effects of preferred orientation are reduced. Also, crystal structure and microstructure parameters can be simultaneously refined. So, it is a successful approach to determine the degree of crystallinity and to give useful structural picture of crystalline and amorphous counterpart at the same time.

\section{References}

1. C. Wright, "Neutron and X-ray Amorphography", Chapter 8 of: Experimental Techniques of Glass Science, Edt. C. J. Simmons and O. H. El-Bayoumi, Ceramic Transactions, American Ceramic Society, pp 205314 (1993).

2. Le Bail, C. Jacobni and R. Pape, J. Phys. Coll. C8, 163 (1985).

3. Le Bail, J. Non-Cryst. Solids 183, 39 (1995).

4. C. Wrigh, J. Non-Cryst. Solids 123, 129 (1990).

5. R. J. Hill and C. J. Howard, J. Appl. Cryst. 20, 467 (1987).

6. E. M. Abdel-Moneim, M. Sc. Thesis, Fac. Science, El-Minia Univ., (1996).

7. A. A. Ramadan, S. K. Abdelraheem, M. R. Ebeid and E. M. AbdelMoneim, XVII Conf. on Appl. Cryst., Abs. p.64 (1997).

8. L. Lutterotti, R. Ceccato, R. D. Maschis and E. Paganie, Mat. Sc. Forum 278, 87 (1998).

9. M. R. Ebeid, S. K. Abdelraheem, E. M. Abdel-Moneim, K. Abdel-Hady and A. A. Ramadan, Egypt. J. Solids 23, 1 (2000).

10. P. Scardi, L. Lutterotti and P. Maistrelli, J. Appl. Cryst. 25, 459 (1992).

11. P. H. Gaskell, and D. J. Wallis, Phys. Rev. Lett. 76, 66 (1996).

12. H. M. Rietveld, J. Appl. Cryst. 2, 65 (1969). 
13. R. L. McGreevy and L. Pusztai, Mol. Simulation, 1, 359 (1988).

14. D. A. Keen and R. L. McGreevy; Nature 344, 423 (1990).

15. D. A. Keen and M. T. Dove, J. Phys.: Condens. Matter 11, 9263 (1999).

16. R. W. G. Wyckoff, "Crystal Structures", John Willey \& Sons, Inc., New York, (1963).

17. J. H. Wakelin, H. S. Virgin and E. Crystal, Appl. Phys. 30, 1954 (1959).

18. W. O. Satton, J. Appl. Polm. Sci. A2, 10, 1407 (1972).

19. S. Barrett and T. B. Massalski, "Structure of Metals", McGraw-Hill Series in Materials Science and Engineering, p. 57 (1966). 